\title{
REGENERAÇÃO NATURAL EM UM REMANESCENTE DE CAATINGA COM DIFERENTES HISTÓRICOS DE USO NO AGRESTE PERNAMBUCANO ${ }^{1}$
}

Shirley de Oliveira Silva ${ }^{2}$, Rinaldo Luiz Caraciolo Ferreira ${ }^{3}$, Jose Antonio Aleixo da Silva ${ }^{3}$, Mario de Andrade Lira ${ }^{4}$, Francisco Tarcísio Alves Junior ${ }^{3}$, Maria Olivia de Oliveira Cano ${ }^{4}$ e José Edson Lima Torres ${ }^{5}$

\begin{abstract}
RESUMO - O objetivo deste trabalho foi comparar a regeneração natural de duas áreas de vegetação de caatinga com diferentes históricos de uso no agreste pernambucano. O estudo foi realizado em duas áreas: Área I de mata nativa, sem evidências históricas de eliminação total da vegetação para fins de cultivos agrícolas; e Área II, anteriormente ocupada com cultivo da palma forrageira, abandonada há cerca de 30 anos e que se encontrava em estágio de sucessão secundária. Para coleta dos dados da vegetação, foram utilizadas 24 parcelas de dimensões de 5 x $5 \mathrm{~m}$, em 12 parcelas em cada área, sendo considerados indivíduos em regeneração natural as plantas que possuíram circunferência a $1,30 \mathrm{~m}$ do solo (CAP) $<6,0 \mathrm{~cm}$ e altura $\geq 1,0 \mathrm{~m}$. No levantamento estrutural dos indivíduos regenerantes das duas áreas foram identificados 581 plantas pertencentes a 14 famílias botânicas, 26 gêneros e 30 espécies. As densidades totais obtidas neste estudo foram de 11.200 ind.ha $^{-1}$ e 8.116 ind.ha $^{-1}$, nas Áreas I e II, respectivamente. As espécies que obtiveram maiores densidades de regeneração natural na Área I foram: Croton argyrophyllus, Senegalia bahiensis, Croton blanchetianus e Coutarea hexandra; e na Área II: Senegalia bahiensis, Poincianella pyramidalis, Zanthoxyllum sp, Croton blanchetianus e Croton argyrophyllus.

Palavras-chave: São Bento do Una, Níveis de perturbação e Sucessão ecológica.
\end{abstract}

\section{ADVANCED NATURAL REGENERATION IN A CAATINGA REMAINDER WITH DIFFERENT DESCRIPTIONS OF USE IN THE AGRESTE OF PERNAMBUCO, BRAZIL}

\begin{abstract}
The objective of this work was to compare a natural regeneration of two areas with Caatinga vegetation with different histories of use in the Agreste of Pernambuco. These studies were carried out in two areas: Area l, native forest, with no historical evidences of deforestation for agriculture purposes; and Area ll, which was previously occupied with the cultivation of forage cactus and was abandoned about 30 years ago at the stage of secondary succession. For data collection, it was used a total of 24 plots of $5 x$ $5 \mathrm{~m}$ dimensions, 12 plots in each area, and considering the plants with circumference at breast height $(\mathrm{CBH})$ of $1.30 \mathrm{~m}$ from ground $(C A P)<6.0 \mathrm{~cm}$ and height $\geq 1.0 \mathrm{~m}$ as individuals in natural regeneration. It was identified in the structure survey of regenerating individuals structural survey in both areas, 581 plants of 14 botanical families, 26 genus and 30 species. The total densities obtained in this study were 11,200 and 8,116 individuals.ha-1 in Area l and ll, respectively. The species with the greatest densities of natural regeneration in Area l were the following: Croton argyrophyllus, Senegalia bahiensis, Croton blanchetianus and Coutarea hexandra and in the Area ll: Senegalia bahiensis, Poincianella pyramidalis, Zanthoxyllum sp, Croton blanchetianus and Croton argyrophyllus.
\end{abstract}

Keywords: São Bento do Una, Levels of disturbance and Ecological succession.

\footnotetext{
${ }^{1}$ Recebido em 16.12.2008 e aceito para publicação em 19.04.2012.

${ }^{2}$ Centro Universitário de Caratinga, UNEC, Brasil. E-mail: <shirleyoliveira10@yahoo.com.br>.

${ }^{3}$ Universidade Federal Rural de Pernambuco, UFRPE, Brasil. Email: <rinaldo@dcfl.ufrpe.br>, <aleixo@dcfl.ufrpe.br>e $<$ tarcisioalvesjr@yahoo.com.br>.

${ }^{4}$ Empresa Pernambucana de Pesquisa Agropecuaria, IPA, Brasil. E-mail: <mariolira@terra.com.br>e <ipa@ipa.br>.

${ }^{5}$ Pós Graduação em Engenharia Civil e Ambiental pela Universidade Federal de Pernambuco, UFPE, Brasil. E-mail: <edsonfloresta@yahoo.com.br>.
} 


\section{INTRODUÇÃO}

A distribuição da vegetação no Nordeste do Brasil é influenciada pelo gradiente climático, seguindo-se a diminuição na média pluviométrica do litoral em direção ao interior do continente, onde a vegetação perde em altura e ganha em espécies decíduas e sua fisionomia toma contornos de floresta seca, conhecida regionalmente como caatinga (VICENTE et al., 2005).

Alterações na caatinga tiveram início com o processo de colonização do Brasil, inicialmente como consequência da pecuária bovina, associada às práticas agrícolas rudimentares. Não obstante, a caatinga possui resistência às perturbações antrópicas, como os processos de corte e queima sistematicamente aplicados em muitas áreas de seu domínio (FREITAS et al., 2007).

O semiárido brasileiro é considerado a região árida mais habitada do mundo. Contudo, a pressão antrópica vegetação da caatinga vem se intensificando ao longo dos anos, principalmente a partir do corte indiscriminado de espécies arbóreas nativas (DRUMOND et al., 2008).

As explorações excessivas dos recursos naturais da caatinga com o alto nível de devastação da vegetação nativa vêm provocando impactos ambientais de grande magnitude, cujas consequências exigem intervenção imediata no sentido de amenizar os problemas daí decorrentes (PEREIRA et al., 2002).

A utilização racional e permanente dos recursos florestais de qualquer ecossistema só pode ser planejada a partir do conhecimento de suas dinâmicas biológicas, tornando-se imperativo conhecer, por exemplo, como se dão os processos de regeneração natural diante das perturbações antrópicas (PEREIRA et al., 2001).

O conhecimento sobre a Caatinga tem sido em muito ampliado, principalmente no que se refere às áreas específicas, como a depressão sertaneja; e áreas sedimentares no Sertão (PEREIRA et al., 2002; LEMOS et al., 2002; ALCOFORADO-FILHO et al., 2003; QUEIROZ et al., 2006; BARBOSA et al., 2007; CEZAR et al., 2007; FREITAS et al., 2007; RODALet al., 2008; FABRICANTE; ANDRADE, 2008). Entretanto, poucos estudos foram publicados sobre a vegetação caducifólia localizada nas áreas transicionais entre as zonas fisiográficas do Sertão e da Mata, a chamada zona do Agreste (PEREIRA et al., 2001; ANDRADE et al., 2007).

Estudos sobre a dinâmica de regeneração natural são de grande interesse científico, no entanto existem escassez e grandes lacunas desses estudos para a vegetação da caatinga pernambucana. Além disso, tais conhecimentos são essenciais para a elaboração e aplicação correta dos planos de manejo e tratamentos silviculturais, permitindo a exploração racional e permanente dos remanescentes.

Dessa forma, este trabalho teve como objetivo comparar a regeneração natural avançada de duas áreas de vegetação de caatinga com diferentes históricos de uso no agreste de Pernambuco.

\section{MATERIAL E MÉTODOS}

\subsection{Caracterização da área}

A pesquisa foi realizada na Estação Experimental de São Bento do Una, pertencente ao Instituto Agronômico de Pernambuco (IPA), situada na microrregião homogênea do Vale do Ipojuca, Agreste, Semiárido de pernambucano.

A localização geográfica é $08^{\circ} 31$ ' 56" de latitude Sul e $36^{\circ} 33^{\prime} 00^{\prime}$ ' de longitude Oeste e altitude de $650 \mathrm{~m}$. O clima, segundo a classificação de Köppen, é do tipo BSh, semiárido, apresentando precipitação média anual de aproximadamente $655 \mathrm{~mm}$ (FARIAS et al., 2000). Os dados climáticos de precipitação e temperatura do período de condução do estudo foram adquiridos na Estação Meteorológica da Estação de Experimental de São Bento do Una (Figura 1).

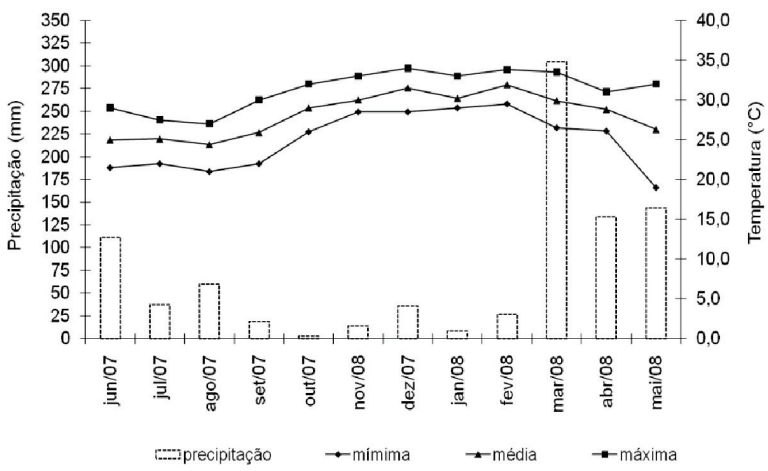

Figura 1 - Precipitação pluviométrica e temperaturas máxima, média e mínima mensal do período de abril/2007 a junho/2008. São Bento do Una, Pernambuco, Brasil.

Figure 1 -Rainfall and maximum, average and minimum monthly temperatures from April/2007 to June/ 2008. São Bento do Una, Pernambuco State, Brazil. 
O estudo foi realizado em duas áreas de caatinga: Área I, com 8,9 ha de área nativa, sem evidências históricas de eliminação total da vegetação para fins de cultivos agrícolas; e Área II, com 9,4 ha, a qual foi anteriormente oocupada com cultivo de palma-forrageira (Opuntia ficus-indica Mill) e abandonada há cerca de 30 anos e que se encontrava em estágio de sucessão secundária. As áreas anteriormente eram apenas uma área contínua, totalizando 18,3 ha, em que foi construída uma estrada de 3 m largura, para separá-las em duas áreas, ficando uma para o cultivo da palma-forrageira e a outra para vegetação. Essas áreas vêm sofrendo ações antrópicas de retirada de madeira ao longo dos anos.

\subsection{Coleta de dados da estrutura da vegetação}

Para coleta dos dados da vegetação foi utilizado um total de 24 parcelas de dimensões de 5 x $5 \mathrm{~m}$, sendo 12 parcelas em cada área e distribuídas de forma sistemática em faixas, cobrindo as áreas de um extremo ao outro, com intervalo de $45 \mathrm{~m}$ entre parcelas na faixa e $65 \mathrm{~m}$ entre faixas.

Foram considerados indivíduos em regeneração natural avançada as plantas que possuíam circunferência a 1,30 $\mathrm{m}$ do solo (CAP) $<6,0 \mathrm{~cm}$ e altura $\geq 1,0 \mathrm{~m}$. Fez-se a inclusão de indivíduos apenas com altura superior a $1 \mathrm{~m}$, para proporcionar identificações mais seguras das espécies e indivíduos que oferecem maior probabilidade de se estabelecer no local, facilitando futuro estudo da dinâmica na área.

Cada indivíduo amostrado recebeu uma etiqueta de PVC numerada em ordem crescente. A altura foi estimada com o auxílio de uma régua numerada de $2 \mathrm{~m}$ de altura, conforme protocolo de medições de parcelas permanentes (Comitê Técnico Científico da Rede de Manejo Florestal da Caatinga, 2005).

Os regenerantes foram divididos em três classes de tamanho de regeneração natural de acordo com Finol (1971) e adaptados às condições da caatinga: Classe I - Indivíduos com altura de 1,0 a 2,0 m; Classe II Indivíduos com altura de 2,01 a 3,0 m; e Classe III Indivíduos com altura superior a 3,0 m.

O material botânico foi identificado inicialmente no local por seu nome vulgar e coletado para confecção de exsicatas. O material foi identificado por especialista botânico e por comparação com exsicatas no Herbário Dárdano de Andrade Lima (IPA) e no Herbário Professor Sérgio Tavares (UFRPE), onde estão depositadas. A sinonímia e grafia dos taxa foram realizadas mediante consulta a Forzza et al. (2010). Para separação em famílias, foi adotado o sistema de classificação APG III (2009).

Para determinação da suficiência do número de parcelas amostradas, utilizou-se o procedimento REGRELRP, do Sistema para Análise Estatística e Genética (SAEG), desenvolvido pela Universidade Federal de Viçosa, conforme adotado por Ferreira e Vale (1992), seguindo-se a lógica da curva espécie/área. Esse procedimento é apropriado para análise de regressão de modelos descontínuos, compostos de uma parte linear crescente e de uma na forma de platô (SAEG, 1997).

Foram calculados parâmetros estruturais de acordo com Mueller-Dombois e Ellemberg (1974). Para estimar a diversidade florística, foi utilizado o índice diversidade de Shannon-Wiener $\left(\mathrm{H}^{\star}\right)$, conforme descrito em Magurran (1988). Os dados foram processados com o auxílio do software Mata Nativa 3 (CIENTEC, 2010).

\section{RESULTADOS E DISCUSSÃO}

A amostragem dos dados florísticos, em ambas as áreas, mostrou-se satisfatória para caracterizar a florística da vegetação em estudo utilizando procedimento REGRELRP do SAEG (Figura 2). Nessa figura, observa-se que houve a estabilização do número de espécies.

No levantamento estrutural dos indivíduos regenerantes das duas áreas foram identificadas 581 plantas pertencentes a 17 famílias botânicas, 26 gêneros e 30 espécies, e seis indivíduos não foram identificados devido à falta de material fértil para sua identificação (Tabela 1). Pereira et al. (2001), em uma área com três níveis de perturbação, com $400 \mathrm{~m}^{2}$ cada, no agreste paraibano, encontraram 347 indivíduos no total, representados por 15 famílias, 22 gêneros e 26 espécies.

JáAndrade et al. (2007) também no Estado da Paraíba, estudando campos abandonados de sisal (Agave sisalana Perrine ex Engelm.) há aproximadamente 30 anos, verificaram 797 indivíduos em uma área amostral de $4.000 \mathrm{~m}^{2}$, pertencentes a 15 famílias botânicas, 27 gêneros e 31 espécies; e Fabricante e Andrade (2008), estudando indivíduos regenerantes na caatinga no Seridó paraibano, encontraram os maiores números de indivíduos: 1.129 , pertencentes a 15 espécies, 12 gêneros e sete famílias botânicas.

Revista Árvore, Viçosa-MG, v.36, n.3, p.441-450, 2012 

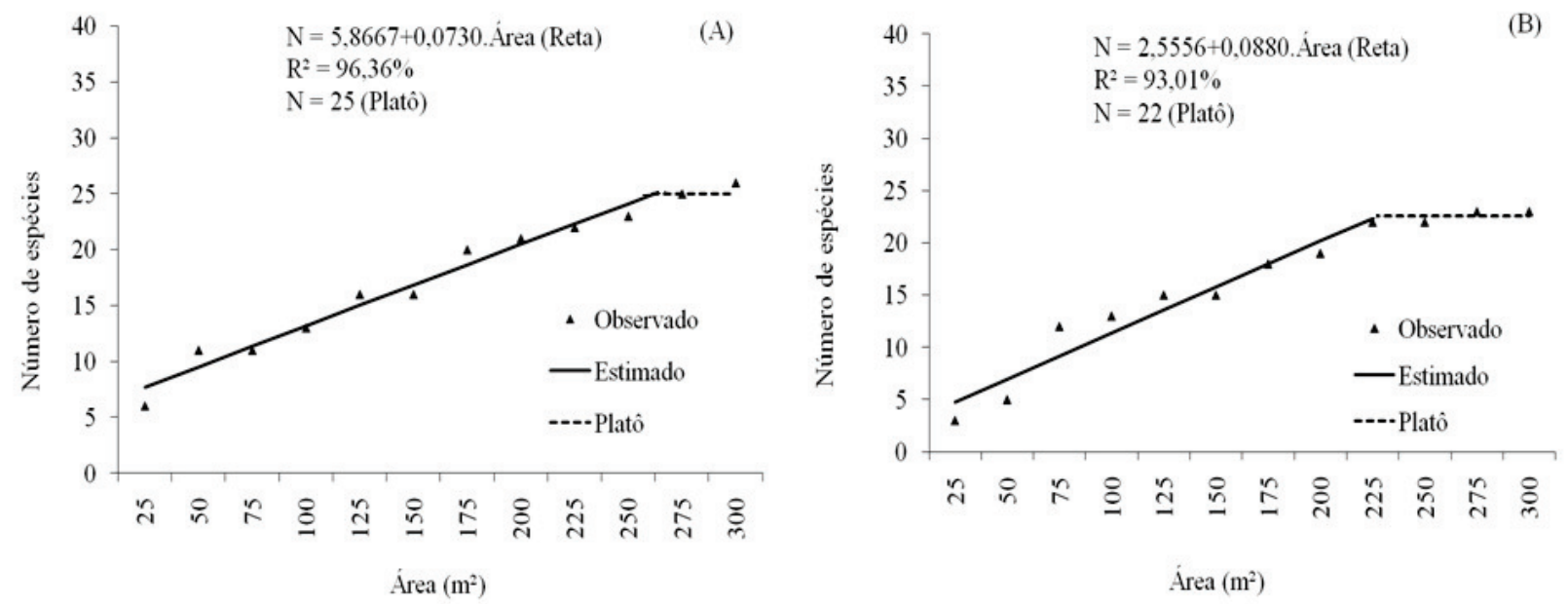

Figura 2 - Representação gráfica da suficiência amostral, área x número de espécies amostradas de duas áreas de caatinga em São Bento do Una, PE. A = Área I e B = Área II.

Figure 2 - Graphical representation of sampling sufficiency, area x number of sampled species in two Caatinga areas in São Bento do Una, Pernambuco State, Brazil. A = Area I and B = Area II.

As variabilidades ocorrentes nos levantamentos florísticos, quanto à quantidade de espécies e indivíduos, levaram Andrade-Lima (1981) a afirmar que as caatingas situadas em locais onde as precipitações são mais acentuadas apresentam maior número de espécies. Entretanto, Rodal et al. (2008) relataram que não apenas o total de chuvas pode modificar a quantidade de espécies e indivíduos, embora esse seja um dos fatores mais importantes, elegendo outros elementos a serem considerados, como situação topográfica, classe, profundidade e permeabilidade do solo.

Além dos fatores edafoclimáticos em que esses remanescentes estão inseridos, deve-se levar em consideração o nível de inclusão adotado, que em muitos estudos é diferenciado. Santana e Souto (2006) afirmaram que áreas que sofrem antropismo devem levar em consideração o histórico de seu uso.

Na Área I, registraram-se 17 famílias botânicas, 21 gêneros e 26 espécies, e três indivíduos não foram identificados. As famílias de maior representatividade foram: Fabaceae com cinco, Euphorbiaceae com quatro, Capparaceae e Erytroxylaceae com dois gêneros. Já as demais famílias foram representadas por apenas uma espécie. As famílias Euphorbiaceae e Fabaceae corresponderam a 50\% do total de espécies da área. Essas famílias também se apresentaram como de maior número de espécies em outras pesquisas sobre regeneração natural (PEREIRA et al., 2001; ANDRADE et al., 2007).

Na Área II, amostraram-se 14 famílias botânicas, 19 gêneros e 23 espécies, e três indivíduos não foram identificados. As famílias que mais se destacaram foram: Fabaceae, com 6 gêneros, Euphorbiaceae com quatro e Capparaceae com dois; as demais famílias foram representadas por apenas uma espécie e detiveram cerca de $52 \%$ do total das espécies amostradas nessa área.

As espécies Schinopsis brasiliensis, Erythroxylum subrotundum, Sapium glandulatum, Senegalia langsdorffii, Prockia crucis e Rhamnidium elaecarpum foram encontradas apenas na Área I, assim como as espécies Ptilochaeta banhiensis e Piptadenia viridiflora, que foram exclusivas da Área II, enquanto as demais espécies foram comuns às duas áreas estudadas.

Analisando o fato de que essas áreas há 30 anos faziam parte de uma área contínua, a afirmação de Pereira et al. (2003) de que a vegetação das áreas de caatinga em regeneração tem estrutura diferente da anterior ao desmatamento, mesmo muitos anos depois, corrobora os resultados deste estudo.

As densidades totais obtidas neste trabalho foram de 11.200 ind.ha $^{-1}$ e 8.116 ind.ha $a^{-1}$, nas Áreas I e II, respectivamente (Tabelas 2 e 3 ). Esses valores foram 
Tabela 1 - Listagem florística das áreas I e II de vegetação de caatinga, em ordem alfabética por família, São Bento do Una, PE. Table 1 - Flower list of areas I and II with Caatinga vegetation caatinga, in alphabetical order of familes, São Bento do Una, Pernambuco State, Brazil.

\begin{tabular}{|c|c|c|c|}
\hline Família/Espécies & Nome vulgar & $\mathrm{I}$ & II \\
\hline \multicolumn{4}{|l|}{ Anacardiaceae } \\
\hline Schinopsis brasiliensis Engl. & Baraúna & $\mathrm{x}$ & \\
\hline \multicolumn{4}{|l|}{ Apocynaceae } \\
\hline Aspidosperma pyrifolium Mart. & Pereiro & $\mathrm{x}$ & $\mathrm{x}$ \\
\hline \multicolumn{4}{|l|}{ Boraginaceae } \\
\hline Varronia globosa Jacq. & Moleque duro & $\mathrm{x}$ & $\mathrm{x}$ \\
\hline \multicolumn{4}{|l|}{ Capparaceae } \\
\hline Capparis flexuosa $(\mathrm{L}) \mathrm{L}$. & Feijão bravo & $\mathrm{x}$ & $\mathrm{x}$ \\
\hline Capparis jacobinae Moric ex Eicheud & Icó & $\mathrm{x}$ & $\mathrm{x}$ \\
\hline \multicolumn{4}{|l|}{ Celastraceae } \\
\hline Maytenus rigida Mart. & Bom nome & $\mathrm{x}$ & $\mathrm{x}$ \\
\hline \multicolumn{4}{|l|}{ Erytroxylaceae } \\
\hline Erythroxylum subrotundum A. St. Hil & Rompe gibão & $\mathrm{x}$ & \\
\hline Erythroxylum cf. macrochaetum Miquel & - & $\mathrm{x}$ & $\mathrm{x}$ \\
\hline \multicolumn{4}{|l|}{ Euphorbiaceae } \\
\hline Croton argyrophyllus Kunth & Velame & $\mathrm{x}$ & $\mathrm{x}$ \\
\hline Croton blanchetianus Baill. & Marmeleiro & $\mathrm{x}$ & $\mathrm{x}$ \\
\hline Croton heliotropiifolius Kunth. & Velame & $\mathrm{x}$ & $\mathrm{x}$ \\
\hline Jatropha mollissima Muell. Arg. & Pinhão bravo & & $\mathrm{x}$ \\
\hline Sapium glandulatum ( Vell.) Pax & Burra leiteira & $\mathrm{x}$ & \\
\hline \multicolumn{4}{|l|}{ Fabaceae } \\
\hline Bauhinia cheilantha (Bong.) Steud. & Mororó & $\mathrm{x}$ & $\mathrm{x}$ \\
\hline Chloroleocon foliolosum (Benth.) J. P. Lewis & & & $\mathrm{x}$ \\
\hline Mimosa tenuiflora (Willd.) Poir. & Jurema preta & $\mathrm{x}$ & $\mathrm{x}$ \\
\hline Piptadenia viridiflora (Kunth) Benth. & Espinheiro & & $\mathrm{x}$ \\
\hline Poincianella pyramidalis (Tul.) L.P.Queiroz & Catingueira & $\mathrm{x}$ & $\mathrm{x}$ \\
\hline Senegalia bahiensis (Benth.) Seigler \& Ebinger & Jurema branca & $\mathrm{x}$ & $\mathrm{x}$ \\
\hline Senegalia langsdorffii (Benth.) Seigler \& Ebinger & Jurema & $\mathrm{x}$ & \\
\hline \multicolumn{4}{|l|}{ Malpighiaceae } \\
\hline Ptilochaeta cf. banhiensis Turcz & - & & $\mathrm{x}$ \\
\hline \multicolumn{4}{|l|}{ Nyctaginaceae } \\
\hline Guapira noxia (Netto) Lund. & Cuiteiro & $\mathrm{x}$ & $\mathrm{x}$ \\
\hline \multicolumn{4}{|l|}{ Rhamnaceae } \\
\hline Rhamnidium elaecarpum Reissek & Cabrito & $\mathrm{x}$ & \\
\hline \multicolumn{4}{|l|}{ Rubiaceae } \\
\hline Coutarea hexandra (Jacq.) K.Schum & Quina-quina & $\mathrm{x}$ & $\mathrm{x}$ \\
\hline \multicolumn{4}{|l|}{ Rutaceae } \\
\hline Zanthoxyllum sp & - & $\mathrm{x}$ & $\mathrm{x}$ \\
\hline \multicolumn{4}{|l|}{ Salicaceae } \\
\hline Prockia crucis $\mathrm{P}$. Browne ex L & - & $\mathrm{x}$ & \\
\hline \multicolumn{4}{|l|}{ Sapindaceae } \\
\hline Allophylus edulis (A. St.-Hil. et al.) Hieron. ex Niederl. & Chal-chal & $\mathrm{x}$ & $\mathrm{x}$ \\
\hline \multicolumn{4}{|l|}{ Solonaceae } \\
\hline Solonaceae 1 & - & $\mathrm{x}$ & $\mathrm{x}$ \\
\hline \multicolumn{4}{|l|}{ Verbenaceae } \\
\hline Lantana camara $\mathrm{L}$. & Chumbinho & $\mathrm{x}$ & $\mathrm{x}$ \\
\hline Indeterminada 1 & & $\mathrm{x}$ & $\mathrm{x}$ \\
\hline
\end{tabular}

Revista Árvore, Viçosa-MG, v.36, n.3, p.441-450, 2012 
Tabela 2 - Análise de regeneração natural da vegetação caatinga (área I), São Bento do Una-PE. CTA = Classe de Tamanho Absoluta; DR = Densidade Relativa; FR = Frequência Relativa; CTR = Classe de Tamanho da Regeneração; e RNR = Regeneração Natural Relativa.

Table 2 - Analysis of Natural Regeneration of Caatinga vegetation (Area I), São Bento do Una, Pernambuco State, Brazil. $C T A=$ Absolute Size Class; DR= Relative Density; FR=Relative Frequency; CTR=Class of Size of Regeneration; and $R N R=$ Relative Natural Regeneration .

\begin{tabular}{|c|c|c|c|c|c|c|c|c|c|c|}
\hline \multirow{2}{*}{ Espécie } & \multicolumn{3}{|c|}{ Classe de Altura } & \multicolumn{3}{|c|}{ CTA } & $\mathrm{DR}$ & FR & $\mathrm{CTR}$ & RNR \\
\hline & I & II & III & $\mathrm{CI}$ & $\mathrm{CII}$ & CIII & \multicolumn{4}{|c|}{$\%$} \\
\hline Croton argyrophyllus & 76 & 69 & 25 & 3211,9 & 2649,11 & 483,63 & 50,6 & 14,46 & 51,96 & 39,01 \\
\hline Senegalia bahiensis & 12 & 14 & 7 & 507,14 & 537,5 & 135,42 & 9,82 & 12,05 & 9,66 & 10,51 \\
\hline Coutarea hexandra & 8 & 5 & 10 & 338,1 & 191,96 & 193,45 & 6,85 & 6,02 & 5,93 & 6,27 \\
\hline Varronia globosa & 11 & 7 & 0 & 464,88 & 268,75 & 0 & 5,36 & 6,02 & 6,01 & 5,80 \\
\hline Croton blanchetianus & 7 & 8 & 4 & 295,83 & 307,14 & 77,38 & 5,65 & 4,82 & 5,57 & 5,35 \\
\hline Aspidosperma pyrifolium & 3 & 6 & 0 & 126,79 & 230,36 & 0 & 2,68 & 8,43 & 2,92 & 4,68 \\
\hline Poincianella pyramidalis & 5 & 3 & 3 & 211,31 & 115,18 & 58,04 & 3,27 & 7,23 & 3,15 & 4,55 \\
\hline Capparis jacobinae & 4 & 2 & 1 & 169,05 & 76,79 & 19,35 & 2,08 & 6,02 & 2,17 & 3,42 \\
\hline Erythroxylum cf. macrochaetum & 3 & 1 & 2 & 126,79 & 38,39 & 38,69 & 1,79 & 3,61 & 1,67 & 2,36 \\
\hline Schinopsis brasiliensis & 1 & 2 & 2 & 42,26 & 76,79 & 38,69 & 1,49 & 2,41 & 1,29 & 1,73 \\
\hline Senegalia langsdorffii & 1 & 1 & 3 & 42,26 & 38,39 & 58,04 & 1,49 & 2,41 & 1,14 & 1,68 \\
\hline Bauhinia cheilantha & 1 & 2 & 1 & 42,26 & 76,79 & 19,35 & 1,19 & 2,41 & 1,13 & 1,58 \\
\hline Allophylus edulis & 1 & 2 & 1 & 42,26 & 76,79 & 19,35 & 1,19 & 2,41 & 1,13 & 1,58 \\
\hline Sapium glandulatum & 2 & 1 & 0 & 84,52 & 38,39 & 0 & 0,89 & 2,41 & 1,01 & 1,44 \\
\hline Indeterminada 1 & 1 & 1 & 1 & 42,26 & 38,39 & 19,35 & 0,89 & 2,41 & 0,82 & 1,37 \\
\hline Croton heliotropiifolius & 1 & 0 & 2 & 42,26 & 0 & 38,69 & 0,89 & 2,41 & 0,66 & 1,32 \\
\hline Zanthoxyllum sp & 1 & 1 & 0 & 42,26 & 38,39 & 0 & 0,6 & 2,41 & 0,66 & 1,22 \\
\hline Solonaceae 1 & 0 & 1 & 1 & 0 & 38,39 & 19,35 & 0,6 & 2,41 & 0,47 & 1,16 \\
\hline Capparis flexuosa & 0 & 0 & 2 & 0 & 0 & 38,69 & 0,60 & 1,20 & 0,32 & 0,71 \\
\hline Erythroxylum subrotundum & 1 & 0 & 0 & 42,26 & 0 & 0 & 0,30 & 1,20 & 0,34 & 0,61 \\
\hline Mimosa tenuiflora & 1 & 0 & 0 & 42,26 & 0 & 0 & 0,30 & 1,20 & 0,34 & 0,61 \\
\hline Guapira noxia & 1 & 0 & 0 & 42,26 & 0 & 0 & 0,30 & 1,20 & 0,34 & 0,61 \\
\hline Lantana camara & 1 & 0 & 0 & 42,26 & 0 & 0 & 0,30 & 1,20 & 0,34 & 0,61 \\
\hline Maytenus rigida & 0 & 1 & 0 & 0 & 38,39 & 0 & 0,30 & 1,20 & 0,31 & 0,60 \\
\hline Prockia crucis & 0 & 1 & 0 & 0 & 38,39 & 0 & 0,30 & 1,20 & 0,31 & 0,60 \\
\hline Rhamnidium elaecarpum & 0 & 1 & 0 & 0 & 38,39 & 0 & 0,30 & 1,20 & 0,31 & 0,60 \\
\hline Total Geral & 142 & 129 & 65 & 6001,19 & 4952,68 & 1257,44 & 100 & 100 & 100 & 100 \\
\hline
\end{tabular}

Classe I - Indivíduos com altura de 1,0 a 2,0 m; Classe II - indivíduos com altura de 2,01 a 3,0 m; e Classe III - indivíduos com altura superior a $3,0 \mathrm{~m}$.

Class I - Individuals at 1.00 to 2,00 m of height; Class II - individuals at 2.10 to $3.00 \mathrm{~m}$ of height; $2.10 \leq 3.00 \mathrm{~m}$; and Class III individuals $3.00 \mathrm{~m}$ height.

superiores aos encontrados nos estudos na Paraíba, onde Pereira et al. (2001) constataram no Município de Areias, no agreste do Estado, 6.750, 5.500 e 5.100 ind.ha $^{-1}$ nos ambientes I, II e III, respectivamente, ordenados em níveis crescentes de perturbação. Entretanto, Fabricante e Andrade (2008), pesquisando no Município de Santa Luzia no Seridó, estimaram 2.822,5 ind.ha-1 .
Neste estudo, a Área I apresentou maior densidade, e isso reflete quanto dessa área foi alterada, deixando espaços e clareiras que auxiliam no aumento da germinação, promovendo, consequentemente, densidades de regenerantes mais elevadas. Para Amorim et al. (2005), as grandes variações de densidade estão ligadas à ocupação do espaço e ao porte das plantas lenhosas.

Revista Árvore, Viçosa-MG, v.36, n.3, p.441-450, 2012 
Tabela 3 - Análise de Regeneração Natural da vegetação caatinga (área II), São Bento do Una-PE. CTA = Classe de Tamanho Absoluta; DR = Densidade Relativa; FR = Frequência Relativa; CTR = Classe de Tamanho da Regeneração; e RNR = Regeneração Natural Relativa.

Table 3 - AAnalysis of Natural Regeneration of Caatinga vegetation (Area II), São Bento do Una, Pernambuco State, Brazil. $C T A=$ Absolute Size Class; $D R=$ Relative Density; $F R=$ Relative Frequency; CTR = Class of Size of Regeneration; and $R N R=$ Relative Natural Regeneration

\begin{tabular}{|c|c|c|c|c|c|c|c|c|c|c|}
\hline \multirow[t]{2}{*}{ Espécie } & \multicolumn{3}{|c|}{ Classe de Altura } & \multicolumn{3}{|c|}{ CTA } & DR & FR & CTR & RNR \\
\hline & I & II & III & CI & CII & CIII & \multicolumn{4}{|c|}{$\%$} \\
\hline Croton argyrophyllus & 21 & 24 & 24 & 694,3 & 724,90 & 881,63 & 28,16 & 12,86 & 28,00 & 23,01 \\
\hline Croton blanchetianus & 19 & 11 & 11 & 628,2 & 332,24 & 404,08 & 16,73 & 8,57 & 16,60 & 13,97 \\
\hline Zanthoxyllum sp & 12 & 10 & 10 & 396,7 & 302,04 & 367,35 & 13,06 & 10,00 & 12,97 & 12,01 \\
\hline Poincianella pyramidalis & 14 & 4 & 8 & 462,9 & 120,82 & 293,88 & 10,61 & 11,43 & 10,68 & 10,91 \\
\hline Senegalia bahiensis & 4 & 5 & 10 & 132,2 & 151,02 & 367,35 & 7,76 & 8,57 & 7,92 & 8,08 \\
\hline Capparis flexuosa & 1 & 1 & 6 & 33,1 & 30,20 & 220,41 & 3,27 & 7,14 & 3,46 & 4,62 \\
\hline Coutarea hexandra & 1 & 2 & 5 & 33,1 & 60,41 & 183,67 & 3,27 & 4,29 & 3,37 & 3,64 \\
\hline Aspidosperma pyrifolium & 2 & 2 & 2 & 66,1 & 60,41 & 73,47 & 2,45 & 4,29 & 2,43 & 3,06 \\
\hline Piptadenia viridifolia & 1 & 2 & 1 & 33,1 & 60,41 & 36,73 & 1,63 & 5,71 & 1,58 & 2,97 \\
\hline Maytenus rigida & 1 & 1 & 3 & 33,1 & 30,20 & 110,2 & 2,04 & 2,86 & 2,10 & 2,33 \\
\hline Allophylus edulis & 1 & 3 & 1 & 33,1 & 90,61 & 36,73 & 2,04 & 2,86 & 1,95 & 2,28 \\
\hline Indeterminada 1 & 0 & 0 & 3 & 0 & 0 & 110,2 & 1,22 & 2,86 & 1,34 & 1,81 \\
\hline Capparis jacobinae & 0 & 3 & 0 & 0 & 90,61 & 0 & 1,22 & 2,86 & 1,11 & 1,73 \\
\hline Erythoxylum cf. macrochaetum & 0 & 2 & 0 & 0 & 60,41 & 0 & 0,82 & 2,86 & 0,73 & 1,47 \\
\hline Jatropha mollissima & 1 & 0 & 2 & 33,06 & 0 & 73,47 & 1,22 & 1,43 & 1,30 & 1,32 \\
\hline Croton heliotropiifolius & 1 & 1 & 1 & 33,06 & 30,20 & 36,73 & 1,22 & 1,43 & 1,22 & 1,29 \\
\hline Ptilochaeta cf. banhiensis & 0 & 0 & 2 & 0 & 0 & 73,47 & 0,82 & 1,43 & 0,89 & 1,05 \\
\hline Guapira noxia & 0 & 0 & 1 & 0 & 0 & 36,73 & 0,41 & 1,43 & 0,45 & 0,76 \\
\hline Varronia globosa & 1 & 0 & 0 & 33,06 & 0 & 0 & 0,41 & 1,43 & 0,40 & 0,75 \\
\hline Solonaceae 1 & 1 & 0 & 0 & 33,06 & 0 & 0 & 0,41 & 1,43 & 0,40 & 0,75 \\
\hline Bauhinia cheilantha & 0 & 1 & 0 & 0 & 30,20 & 0 & 0,41 & 1,43 & 0,36 & 0,73 \\
\hline Chloroleocon foliolosum & 0 & 1 & 0 & 0 & 30,20 & 0 & 0,41 & 1,43 & 0,36 & 0,73 \\
\hline Mimosa tenuiflora & 0 & 1 & 0 & 0 & 30,20 & 0 & 0,41 & 1,43 & 0,36 & 0,73 \\
\hline Total geral & 81 & 74 & 90 & 2677,96 & 2235,10 & 3306,12 & 100 & 100 & 100 & 100,00 \\
\hline
\end{tabular}

Classe I - Indivíduos com altura de 1,0 a 2,0 m; Classe II - Indivíduos com altura de 2,01 a 3,0 m; e Classe III - Indivíduos com altura superior a $3,0 \mathrm{~m}$.

Class I - Individuals at 1.00 to $2.00 \mathrm{~m}$ of height; Class II - Individuals at 2.10 to $3.00 \mathrm{~m}$ of height; $2.10 \leq 3.00 \mathrm{~m}$ of height; and Class III - Individuals over $3.00 \mathrm{~m}$ high.

As espécies que obtiveram maiores densidades e regeneração natural foram: Croton argyrophyllus, Senegalia bahiensis, Croton blanchetianus e Coutarea hexandra, que juntas representam $81,54 \%$ das plantas amostradas; e as espécies Croton argyrophyllus e Croton blanchetianus corresponderam a 56,25\%. Segundo Pereira et al. (2001), essas espécies são comuns em áreas sob grandes perturbações, devido à sua facilidade de reprodução e dispersão, características essas que fazem delas pioneiras típicas da caatinga e que tendem a dominar os primeiros estágios serais.
A espécie Coutarea hexandra é amplamente distribuída no Brasil (LUCENA et al., 2006), em alguns levantamentos da flora da caatinga em Pernambuco (RODAL et al., 1998; ALCOFORADO-FILHO et al., 2003; RODAL et al., 2008). Alguns autores classificaram essa espécie como secundária inicial (SILVA et al., 2003; MARANGON et al., 2007). Neste estudo, a contribuição da espécie foi de aproximadamente $7 \%$ da densidade relativa.

As informações obtidas a partir dos estudos realizados em outras áreas (RODAL et al., 1998; PEREIRA et al., 2001; ALCOFORADO-FILHO et al., 2003; SILVA

Revista Árvore, Viçosa-MG, v.36, n.3, p.441-450, 2012 
et al., 2003; MARANGON et al., 2007; RODAL et al., 2008) ajudaram a inferir que esse remanescente de vegetação caatinga é muito jovem, pois apresenta, na sua maioria, espécies ditas como pioneiras e secundárias iniciais. Na Área II, as maiores densidades e regeneração natural foram das seguintes espécies, em ordem crescente: Senegalia bahiensis, Poincianella pyramidalis, Zanthoxyllum sp, Croton blanchetianus e Croton argyrophyllus. No cômputo geral, elas representam 76,32\% das plantas amostradas, e 68,57\% dos indivíduos apresentados são das espécies pioneiras, típicas de ambientes antropizados: Poincianella pyramidalis, Croton blanchetianus e Croton argyrophyllus, mostrando tolerância a elevados níveis de perturbações (SAMPAIO et al., 1998; PEREIRA et al., 2001; QUEIROZ et al., 2006). Essa tendência também foi manifestada em Senegalia bahiensis $e$ Zanthoxyllum sp.

Na Área I, nota-se que as duas primeiras classes de altura apresentaram densidades semelhantes, com redução acentuada dos indivíduos maiores que 3,0 m. Na Área II, a maioria dos indivíduos (36\%) estava na classe III, comportamento esse que reflete que o antropismo recente foi mais acentuado na Área I, onde ocorreu sua maior concentração, com $42 \%$ dos indivíduos na primeira classe de altura. Segundo Pereira et al. (2001), o incremento dos indivíduos nas maiores classes de tamanho apresenta razão inversa ao nível de perturbação imputado aos ambientes (Figura 3).

As espécies Croton blanchetianus e Croton argyrophyllus se destacaram em todas as classes de tamanho e nas duas áreas estudadas. Comportamento esse esperado em virtude de se tratarem de espécies com características pioneiras de ambientes antropizados da caatinga, como apontaram os resultados obtidos por Pereira et al. (2001).

Conclui-se que as duas áreas estudadas sofreram níveis de perturbações antrópicas acentuadas, e isso refletiu no número elevado relacionado à densidade das espécies colonizadoras e típicas de ambientes antropizados, como Croton blanchetianus e Croton argyrophyllus, em ambas as áreas.

Os resultados da Classe I devem ser interpretados com cautela, já que o critério de inclusão dos indivíduos desprezou os exemplares menores que $1 \mathrm{~m}$.

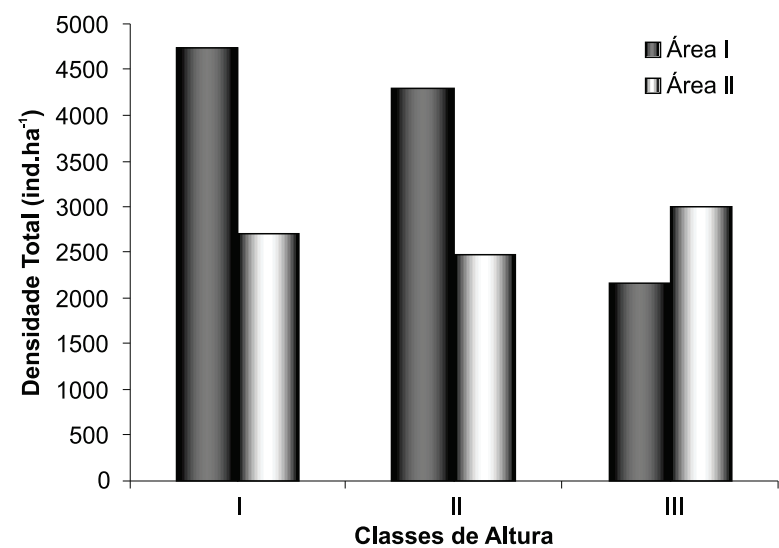

Figura 3 - Distribuição da densidade por classe de tamanho da regeneração natural, em duas áreas de caatinga, São Bento do Una, PE. Classe I - Indivíduos com altura de 1,0 a 2,0 m; Classe II - Indivíduos com altura de 2,01 a 3,0 m; e Classe III - Indivíduos com altura superior a $3,0 \mathrm{~m}$.

Figure 3 -Distribution of the density for size class of natural regeneration in two area of Caatinga in São Bento do Una, Pernambuco State, Brazil. Class I - Individuals at 1.00 to $2.00 \mathrm{~m}$ of height; Class II - Individuals at 2.10 to $3.00 \mathrm{~m}$ of height; 2.10 ? $3.00 \mathrm{~m}$; and Class III - Individuals over $3.00 \mathrm{~m}$ high.

\section{AGRADECIMENTOS}

Ao IPA, pela disponibilidade da área e das instalações para a pesquisa; ao CNPq, pela bolsa de mestrado do primeiro autor, de produtividade do $2^{\circ} 3^{\circ} 4^{\circ}$ autores e a bolsa de PNPD/CAPES/FACEPE do $5^{\circ}$ autor..

\section{REFERÊNCIAS}

ALCOFORADO-FILHO, F. G.; SAMPAIO. E. V. S. B.; RODAL. M. J. N. Florística e fitossociologia de um remanescente de vegetação caducifólia espinhosa arbórea em Caruaru, Pernambuco. Acta Botânica Brasílica, v.17, n.2, p.287-303, 2003.

AMORIM, I. L.; SAMPAIO, E. V. S. B.; ARAÚJO, E. L. Flora e estrutura da vegetação arbustivaarborea de uma área de caatinga do Sérido, RN, Brasil. Acta Botânica Brasílica, v.19, n.3, p.615-623, 2005.

ANDRADE, L. A. et al. Análise da vegetação sucessional em campos abandonados no agreste paraibano. Revista Brasileira de Ciências Agrárias, v.2, n.2, p.135-142, 2007. 
ANDRADE-LIMA, D. The caatingas dominium. Revista Brasileira de Botânica, v.4, n.2, p.149-153. 1981.

ANGIOSPERM PHYLOGENY GROUP-APG III. An update of the Angiosperm Phylogeny Group classification for the orders and families of flowering plants: APG III. Botanical Journal of the Linnean Society, v.161, n.2, p.105-121, 2009.

BARBOSA, M. R. V. et al. Vegetação e flora no Cariri Paraibano. Oecologia Brasiliensis, v.11, n.3, p.313-322, 2007.

CEZAR, A. F. et al. Flora da Serra do Mel RN na Vila Alagoas. Revista Verde de Agroecologia e Desenvolvimento Sustentável, v.1, n.2, p.100-112, 2006.

CIENTEC. Mata Nativa 3. Manual do usuário. Viçosa-MG, 2010."

COMITÊ TÉCNICO CIENTÍFICO DA REDE DE MANEJO FLORESTAL DA CAATINGA. Rede de manejo florestal da Caatinga: protocolo de medições de parcelas permanentes. Comitê Técnico Científico. Recife: Associação Plantas do Nordeste, 2005. Disponível em: <http://www.cnpf.embrapa.br/pesquisa/sispp/ protocolo_RMFC.pdf>. Acesso em: 15 abr. 2007.

DRUMOND, M. A. et al. Produção e distribuição de biomassa de espécies arbóreas no semi-árido brasileiro. Revista Árvore, v.32, n.4, p.665-669, 2008.

FABRICANTE, J. R; ANDRADE, L. A. Análise estrutural de um remanescente de caatinga no Seridó paraibano Oecologia Brasiliensis, v.11, n 3, p.341-349, 2008.

FARIAS, I. et al. Manejo de colheita e espaçamentos da palma-forrageira em consórcio com sorgo granífero, no agreste de Pernambuco. Pesquisa Agropecuária Brasileira, v.35, n.2, p.341-347, 2000.

FERREIRA, R. L. C.; VALE, A. B. Subsídios básicos para o manejo florestal da caatinga. Revista do Instituto Florestal, v.4, parte 2, p.368-375, 1992.

FREITAS, R. A. C. et al. Estudo florístico e fitossociológico do estrato arbustivo-arbóreo de dois ambientes em Messias Targino divisa RN/PB. Revista Verde de Agroecologia e Desenvolvimento Sustentável, v.2, n.1, p.135-147, 2007.
FINOL, U. H. Nuevos parâmetros a considerarse en el analisis estrutural de las selvas vírgenes tropicales. Revista Florestal Venezuelana, v.14, n.21, 29-42, 1971.

FORZZA, R. C. et al. (Org.) Introdução. In: Lista de Espécies da Flora do Brasil. Rio de Janeiro: Instituto de Pesquisas Jardim Botânico do Rio de Janeiro, 2010. 2v.

LEMOS, J. R.; RODAL, M. J. N. Fitossociologia do componente lenhoso de um trecho da vegetação de Caatinga no Parque Nacional Serra da Capivara Piauí, Brasil. Acta Botânica. Brasílica, v.16, n.1, p.23-42, 2002.

LUCENA, J. E. X. et al. Efeito antinociceptivo e antiinfl amatório do extrato aquoso da entrecasca de Coutarea hexandra Schum. (Rubiaceae).

Revista Brasileira de Farmacognosia, v.16, n.1, p.67-72, 2006.

MAGURRAN, A. E. Ecological diversity and its measurement. Princeton: Princeton University Press, 1988. 192p.

MARANGON, L. C. et al. Estrutura fitossociologica e classificação sucessional do componete arbóreo de um frangmento de floresta estacional semidecidual no município de Viçosa, Minas Gerais. Cerne, v.13, n.2, p.208-221, 2007.

MUELLER-DOMBOIS, D.; ELLENBERG, H. Aims and methods of vegetation ecology. New York: John Wiley \& Sons, 1974. 547p.

PEREIRA, I. M. et al. Regeneração natural em um remanescente de caatinga sob diferentes níveis de perturbação, no agreste paraibano. Acta Botânica Brasílica, v.15, n.3, p.431-426, 2001.

PEREIRA, I. M. L. et al. Composição florística e análise fitossociológica do componente arbustivoarbóreo de um remanescente florestal no Agreste Paraibano. Acta Botânica Brasílica, v.16, n.3, p.357-369, 2002.

PEREIRA, I. M. et al. Use-history effects on structure and flora of caatinga. Biotropica, v.35, n.2, p.154-165, 2003.

Revista Árvore, Viçosa-MG, v.36, n.3, p.441-450, 2012 
QUEIROZ, J. A. et al. Análise da estrutura fitossociologica da Serra do Monte, Boqueirão, Paraiba. Revista Biologia e Ciência da Terra, v.6, n.1, p.251-259, 2006.

RODAL, M. J. N. et al. Fitossociologia do componente lenhoso de um refúgio vegetacional no município de Buíque, Pernambuco. Revista Brasileira de Biologia, v.58, n.3, p.517-526.1998.

RODAL, M. J. N.; MARTINS, F. R.; SAMPAIO, E. V. S. B. Levantamento quantitativo das plantas lenhosas em trechos de vegetação de caatinga em Pernambuco. Caatinga, v.21, n.3, p.192-205, 2008.

SISTEMA PARA ANÁLISE ESTATÍSTICA E GENÉTICA - SAEG. Manual de uso Viçosa, MG: UFV-Funarbe, 1997. 150p.
SAMPAIO, E. V. S. B. et al. Regeneração da vegetação de caatinga após corte e queima em Serra Talhada, PE. Pesquisa Agropecuária Brasileira, v.33, n.5, p.621-632, 1998.

SANTANA, J. A. S.; SOUTO, J. S. Diversidade e estrutura fitossociológica da caatinga na estação ecológica do Seridó-RN. Revista de Biologia e Ciências da Terra, v.6, n.2, p.232-242, 2006.

SILVA, A. F. et al. Composição florística e grupos ecológicos das espécies de um trecho de floresta semidecídua submontana da fazenda São Geraldo, Viçosa-MG. Revista Árvore, v.27, n.3, p.311-319, 2003.

VICENTE, A.; SANTOS, M. M.; TABARELLI, M. Variação do modo de dispersão de espécies lenhosas em um gradiente de precipitação entre floresta seca e úmida no nordeste do Brasil. In: LEAL, I. R.; TABARELLI, M.; SILVA, J. M. C. (Org.). Ecologia e conservação da caatinga. 2.ed. Recife: UFPE, 2005. p.565-592. 